\title{
Structural Optimization of a Wearable Deep Body Thermometer: From Theoretical Simulation to Experimental Verification
}

\author{
Ming Huang,, Toshiyo Tamura, ${ }^{2}$ Zunyi Tang, ${ }^{2}$ Wenxi Chen, ${ }^{3}$ and Shigehiko Kanaya ${ }^{1}$ \\ ${ }^{1}$ Graduate School of Information Science, Nara Institute of Science and Technology, Ikoma 6300192, Japan \\ ${ }^{2}$ Department of Biological Engineering, Osaka Electro-Communication University, Shijonawate, Japan \\ ${ }^{3}$ Biomedical Information Technology Laboratory, The University of Aizu, Tsuruga, Aizuwakamatsu, Japan \\ Correspondence should be addressed to Ming Huang; alex-mhuang@is.naist.jp
}

Received 15 May 2015; Revised 24 September 2015; Accepted 29 September 2015

Academic Editor: Junhang Dong

Copyright (C) 2016 Ming Huang et al. This is an open access article distributed under the Creative Commons Attribution License, which permits unrestricted use, distribution, and reproduction in any medium, provided the original work is properly cited.

\begin{abstract}
Deep body temperature (DBT) has yet to be measured continuously in everyday life, even though it is useful in physiological monitoring and chronobiology studies. We tried to address this issue by developing a transcutaneous thermometer based on the dual-heat-flux method (DHFM) invoking the principle of heat transfer, for which measurement error was mitigated by elaborate design. First, a structural modification based on the original design of the DHFM was implemented by the finite element method. Based on the results of the simulations, prototypes were then implemented and tested with an experimental system that mimicked the thermometer being applied to skin. The simulation phase proposed the adoption of an aluminum cover to boost measurement accuracy and suggested that thermometers of different height be chosen according to specified requirements. The results of the mock-up experiments support the modification put forward in the simulation phase: the standard type ( $15 \mathrm{~mm}$ in height) achieved the accuracy with error below $0.3^{\circ} \mathrm{C}$ while the thin type $\left(9 \mathrm{~mm}\right.$ in height) attained accuracy with error less than $0.5^{\circ} \mathrm{C}$ under normal ambient temperature ranging from 20 to $30^{\circ} \mathrm{C}$. Even though the design should also be examined in vivo, it is believed that this study is an important step in developing a practical noninvasive deep body thermometer.
\end{abstract}

\section{Introduction}

Deep body temperature (DBT) is one of the vital signs for human beings and is typically referred to as the temperature of the natural cavities, for example, the abdomen and the thorax. Strictly speaking, DBT can only be measured by invasive methods such as catheter insertion into rectum [1].

However, noninvasive methods are more acceptable and are therefore more widely used. They can provide approximation to the DBT, which is the temperature at a certain depth under the skin reflecting the real DBT. One good alternative to the invasive methods is the zero-heat-flux method [2], which was improved by Togawa's group [3] and implemented in the CoreTemp medical device (Terumo, Tokyo, Japan). It showed good agreement with distal esophageal temperature [4] and pulmonary blood temperature [5]. Developed with an inlaid heater, this device is considered to be stable (robust to changes in the ambient environment) and sensitive [5]. On the other hand, the heater consumes considerable power, which makes this kind of device only applicable indoors, mainly in hospitals during therapy.

However, continuous DBT measurement is necessary in situations such as heat strain monitoring [6] and female menstrual cycle management [7] and in the treatment of sleep disorders [8] for the estimation of biorhythms. These needs could be met by wearable devices. We suggest that the display and data accumulating functions could be allocated to a smartphone/watch, with the data further analyzed and modeled on a server. In the initial stage of signal extraction, a noninvasive thermometer with low power consumption is indispensable.

More often, it is the temperature fluctuations rather than absolute temperature readings that interest us in the healthcare domain. However, the changes in readings by the devices 


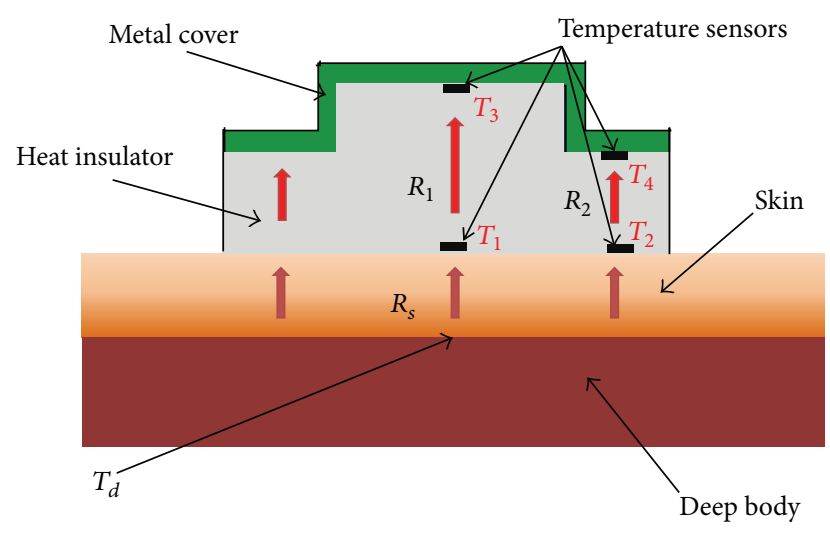

FIGURE 1: Illustration of dual-heat-flux method. The heat flows from deep body into the thermometer longitudinally and thus the DBT can be calculated with the four inlaid sensors $\left(T_{1}-T_{4}\right)$ theoretically.

should be caused by actual changes in the physiological state and not by some external influence. From this viewpoint, an ear-inserted thermometer is not a good choice because its measurements are evidently influenced by the ambient environment. The infrared tympanic thermometer is able to reflect internal change but is sensitive to its positioning.

The mechanism of the thermometer should also be universal; it should be applicable to different people. More specifically, even in the same person, the thermal conductivity of the skin might differ temporally and spatially. A thermometer based on the exact value of the personal physiological condition [9] should be further generalized.

The above concerns can be addressed properly by the dual-heat-flux method (DHFM) [10], the mechanism of which is depicted in Figure 1. The DHFM uses at least four temperature sensors to calculate the DBT, based on the idealized situation that heat flows from the human body into the thermometer longitudinally. This assumption would bring about error in the temperature calculation; however, it requires little information from the user. Moreover, the idealized situation can be approximated by dimensional or thermophysical modification [11].

The criterion of accuracy for clinical use is $0.1^{\circ} \mathrm{C}$ conventionally, which is not readily met by noninvasive methods $[9$, 11]. Hence, we considered $0.5^{\circ} \mathrm{C}$ as the margin for acceptable accuracy in this study. However, for the original design, this level of accuracy is achieved at the cost of a device that is too large to wear or an ultrathin design that is difficult to implement [11]. On the basis of the above understanding, this study aimed to improve the structural design to achieve this level of accuracy without compromising its small size. To reach this target, we first designed and examined the structural modification based on the finite element method (FEM). Then, the validated new structures were fabricated and their capabilities were examined by mock-up experiments.

\section{Materials and Method}

2.1. About DHFM. At least four inlaid temperature sensors are necessary for a thermometer based on DHFM. They are $T_{1}-T_{4}$ in Figure 1. On account of temperature gradient, heat flow arises and flow through the skin layer into the thermometer as shown by red arrows. $T_{d}$ is the general DBT beneath the skin and subcutaneous layer. Assuming that the heat flow from the deep body into the thermometer remains the same, Fourier's law applies, giving

$$
\begin{aligned}
& \frac{\left(T_{d}-T_{1}\right)}{R_{s}}=\frac{\left(T_{1}-T_{3}\right)}{R_{1}}, \\
& \frac{\left(T_{d}-T_{2}\right)}{R_{s}}=\frac{\left(T_{2}-T_{4}\right)}{R_{2}} .
\end{aligned}
$$

$T_{1}$ and $T_{2}$ are skin temperatures measured by two cutaneous temperature sensors inside the thermometer. $T_{3}$ and $T_{4}$ are the temperatures measured by the other two sensors. $R_{s}$ is the heat resistance of the skin, with $R_{1}$ and $R_{2}$ being the heat resistance of the two heat paths inside the thermometer. According to (1), $T_{d}$ then can be expressed as [10]

$$
T_{d}=T_{1}+\frac{\left(T_{1}-T_{2}\right)\left(T_{1}-T_{3}\right)}{k\left(T_{2}-T_{4}\right)-\left(T_{1}-T_{2}\right)} .
$$

Hence, $k=R_{1} / R_{2}$, and it can be represented as the ratio of the heights of the two concentric cylinders used in the thermometer fabrication.

Generally speaking, DBT is strictly regulated by hypothalamus and it changes tardily in accordance to biorhythm. These characteristics make the measurement with DHFM applicable. For such a passive method with heat insulator as the substrate material, it takes time for initial response.

2.2. Simulation Based on FEM. Bioheat transfer involves blood perfusion and metabolic processes. It can be well described by the Pennes equation [12]:

$$
\begin{aligned}
\rho c_{p} \frac{\partial T(\mathbf{X}, t)}{\partial t}= & \nabla \cdot[k(\mathbf{X}) \nabla T(\mathbf{X}, t)] \\
& +\omega_{b} \rho_{b} c_{b}\left(T_{b}-T(\mathbf{X}, t)\right)+q_{m}(\mathbf{X}, t) \\
& \quad \mathbf{X} \in \Omega_{T} .
\end{aligned}
$$

The parameters $T, \rho, c_{p}$, and $k$ are the local temperature, the density $\left(\mathrm{kg} / \mathrm{m}^{3}\right)$, the specific heat $\left(\mathrm{J} / \mathrm{kg} \cdot{ }^{\circ} \mathrm{C}\right)$, and the thermal conductivity $\left(\mathrm{W} / \mathrm{m} \cdot{ }^{\circ} \mathrm{C}\right)$ of the local tissue, respectively. $T_{b}, \omega_{b}$, $\rho_{b}$, and $c_{b}$ are the blood temperature, blood perfusion rate $\left(\mathrm{m}^{3} / \mathrm{m}^{3} \cdot \mathrm{s}\right)$, blood density $\left(\mathrm{kg} / \mathrm{m}^{3}\right)$, and the specific heat of blood $\left(\mathrm{J} / \mathrm{kg} \cdot{ }^{\circ} \mathrm{C}\right)$, respectively.

The second and third terms on the right denote the heating effect of blood perfusion and metabolism. However, these two terms were not considered in this model because this kind of evenly distributed heat source would alter the actual value of the measurement somewhat but would not invalidate the qualitative relations from the simulation. Further, because the DBT should vary in a quasistatic manner, the temporal change was not considered.

Because we assumed that the thermometer and skin were covered by suitable clothing, heat convection was neglected. However, radiation was inevitable; therefore, the boundary 


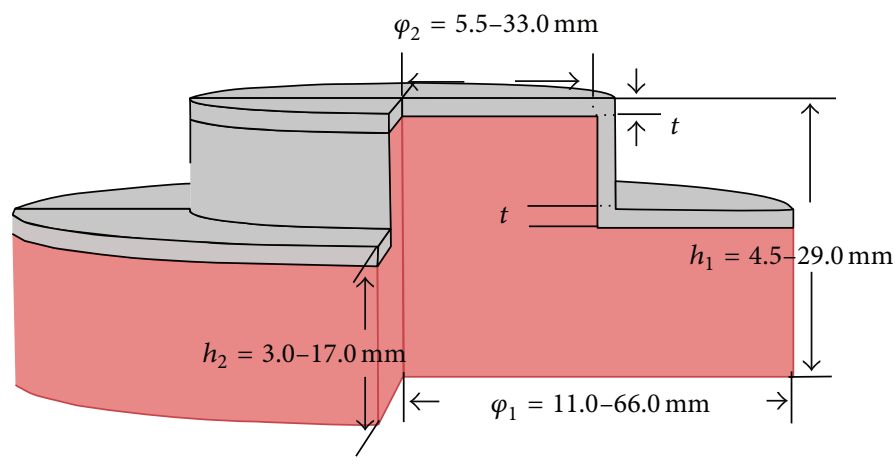

(a)

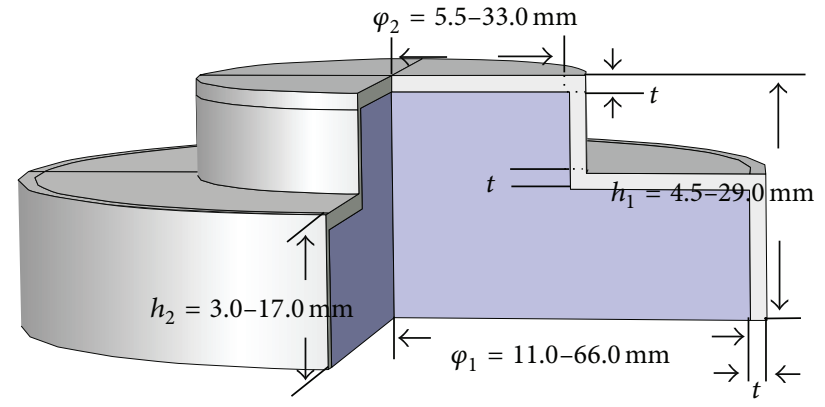

(b)

FIGURE 2: Sectional view of two models. The original structure is without PAR (a), while the modified structure with PAR is shown by (b).

conditions of the thermometer and its surrounding skin could be described by the Stefan-Boltzmann law [13]:

$$
-\overrightarrow{\mathbf{n}} \cdot[-k(\mathbf{X}) \nabla T(\mathbf{X}, t)]=\varepsilon \sigma\left(T_{\mathrm{amb}}^{4}-T_{\mathrm{boun}}^{4}(\mathbf{X}, t)\right),
$$

$$
X \in \Gamma
$$

where $T_{\mathrm{amb}}$ is the ambient temperature, $T_{\text {boun }}$ the local temperature value at the boundary, $\sigma$ the Stefan-Boltzmann constant, and $\varepsilon$ the emissivity of the boundary.

In the simulation phase, we sought to improve the thermometer's accuracy by structural modification, while reserving the wearability of the thermometer. Therefore, an additional component, a peripheral aluminum ring (PAR), was introduced in view of the high emissivity of the insulator component. There were two kinds of thermometers in this simulation, with and without the PAR, as shown in Figures 2(a) and 2(b).

As shown in Figure 2, we considered four combinations of heights, namely, $(29.0,17.0),(15.0,9.0),(8.0,5.0)$, and (4.5, $3.0)$, for $\left(h_{1}, h_{2}\right)$. Similarly, we considered six combinations of radii, namely, $(66.0,33.0),(55.0,27.5),(44.0,22.0),(33.0$, $16.5),(22.0,11.0)$, and $(11.0,5.5)$, for $\left(\varphi_{1}, \varphi_{2}\right)$, respectively. (We also labeled these configurations in terms of their $h_{1}$ and $\varphi_{1}$ values.) In fact, because a radius larger than $50.0 \mathrm{~mm}$ is not suited for wearable application, we introduced $66.0 \mathrm{~mm}$ and $55.0 \mathrm{~mm}$ sizes just for the whole picture of the relation between radius and accuracy. In total, there were 24 dimensional combinations, all of which were simulated by our models. In Figure 2(b), the PAR made of aluminum was represented in silver with $t=1.0 \mathrm{~mm}$, a size that will not bring about distinct change in volume and weight. The ambient temperature of the simulation was $T_{\mathrm{amb}}=25.0^{\circ} \mathrm{C}$ and $T_{d}=37.0^{\circ} \mathrm{C}$. The thickness of the skin and subcutaneous layer was $10.0 \mathrm{~mm}$.

In this model, the rubber was used as insulator and the aluminum was used as the metal. Necessary physical parameters are tabulated in Table 1 . The models were built, simulated, and analyzed with LiveLink for MATLAB based on COMSOL Multiphysics 4.3a (COMSOL Inc., Stockholm, Sweden). Unconstructed mesh was generated by COMSOL adapting to the current physics and geometries. The numbers of elements were different across the models (e.g., 57544
TABLE 1: Thermal properties of various materials*.

\begin{tabular}{lcccc}
\hline Component & $\begin{array}{c}\text { Conductivity } \\
\left(\mathrm{W} / \mathrm{m} \cdot{ }^{\circ} \mathrm{C}\right)\end{array}$ & $\begin{array}{c}\text { Density } \\
\left(\mathrm{kg} / \mathrm{m}^{3}\right)\end{array}$ & $\begin{array}{c}\text { Specific heat } \\
\left(\mathrm{J} / \mathrm{kg} \cdot{ }^{\circ} \mathrm{C}\right)\end{array}$ & Emissivity \\
\hline Skin & 0.17 & 1100 & 3500 & 0.98 \\
Rubber & 0.06 & 180 & 2010 & 0.95 \\
Aluminum & 400 & 8700 & 385 & 0.05 \\
\hline
\end{tabular}

${ }^{*}$ The properties used here were cited from the materials library of COMSOL Multiphysics.

for standard type model); however, the minimum element quality $(q)$ was kept bigger than 0.1. $q$ has a considerable impact on accuracy of the solution, and, for 3D model, the mesh quality should not affect the solution's quality if $q>0.1$.

In practical implementation, the values of the parameters or even the specified materials may be different. The lower the conductivity of the insulator, the higher the accuracy at the cost of a longer initial response in general. The simulation here serves as an examination of the new design.

2.3. Fabrication of Prototypes. Based on the results of the simulation, we fabricated two prototypes of different heights, $h_{1}=9.0 \mathrm{~mm}$ (thin type) and $h_{1}=15.0 \mathrm{~mm}$ (standard type), as shown in Figure 3. Both prototypes were of the same radius, $\varphi_{1}=22.0 \mathrm{~mm}$. The prototypes consisted of two parts: the probe to be applied to the skin for measurement and the processing circuit. As designed in the simulation phase, the probe consists of a heat resistor made of chloroprene rubber and metal components made of aluminum 2017 alloy. Miniature digital temperature sensors LM73 (11-14 bits; Texas Instruments, Dallas, TX) were used as the four inlaid sensors and communicated with the central board with $\mathrm{I}^{2} \mathrm{C}$.

The probe of the thermometer connects to the main processing board by USB cable (Mini to Standard plug). The main processing board consists mainly of the controlling unit (ATmega164A, Atmel RISC Microcontroller; Atmel, San Jose, $\mathrm{CA}$ ), a memory unit with $8 \mathrm{Mb}$ serial flash memory to store the infradian data, and a battery unit to supply three days of energy for the whole local system. Data stored locally can be retrieved with a specialized program run on the PC as a CSV file. 


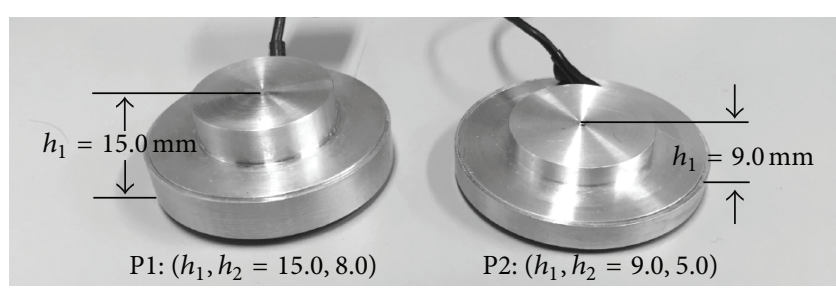

Figure 3: Prototypes of thermometers based on DHFM. Line-up of the standard type and thin type is shown.

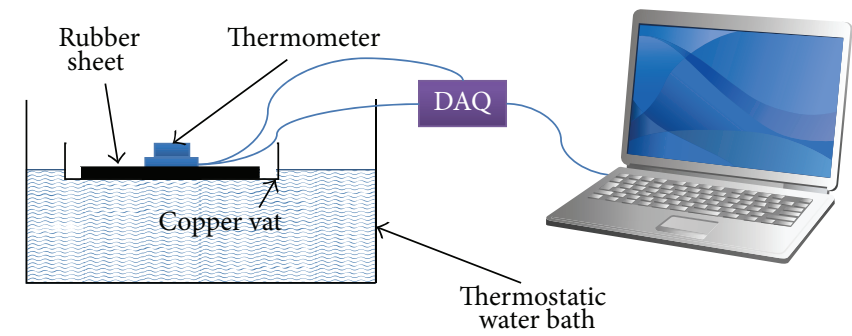

FIGURE 4: Illustration of the mock-up experiment system. The temperature of the water was used to mimic the DBT, while the natural rubber was used to mimic the skin layer.

2.4. Mock-Up Experiment. The structures suggested by the simulation phase should be validated by mock-up experiments before being applied in practical measurement. A standard experimental system $[10,14]$ was used to examine the thermometer's capability. An illustration of the system is shown in Figure 4. The thermometer was placed on a natural rubber sheet (sheets of 2.0, 4.0, 6.0, 8.0, and $10.0 \mathrm{~mm}$ in thickness), which was laid inside a copper vat. The vat floated on temperature-controlled water. In view of the excellent heat conductivity of copper, the boundary between the rubber sheet and copper vat was considered isothermal. Silicon grease was applied to the boundary between the probe and the rubber sheet to minimize thermal resistance. The temperature of the water inside a thermostatic water bath (FR-004, $\pm 0.1^{\circ} \mathrm{C}$; TGK, Tokyo, Japan) was used to mimic $T_{d}$, while the natural rubber sheet was used to mimic human skin, based on the physical similarities of these two materials.

The prototypes were tested in three conditions: without PAR (N), with PAR (A), and without PAR but with sponge cover (S). Through this phase, we hoped to find out whether the new component (i.e., the PAR) would influence the measurements as predicted by the simulation. Condition $S$ was adopted to confirm the effect of the sponge in measuring accuracy; although it is obstructive in practical measurement, it is reportedly indispensable [10].

The range of normal $T_{d}$ falls in between $36.0^{\circ} \mathrm{C}$ and $38.0^{\circ} \mathrm{C}$, while normal indoor $T_{\mathrm{amb}}$ is $20.0-30.0^{\circ} \mathrm{C}$, which may influence the measurements. Thus, we further tested the prototypes in the following three combinations:

$$
\begin{aligned}
& \text { Combination 1: } T_{\mathrm{amb}}=20.0^{\circ} \mathrm{C}, T_{d}=36.0^{\circ} \mathrm{C} . \\
& \text { Combination 2: } T_{\mathrm{amb}}=25.0^{\circ} \mathrm{C}, T_{d}=37.0^{\circ} \mathrm{C} . \\
& \text { Combination 3: } T_{\mathrm{amb}}=30.0^{\circ} \mathrm{C}, T_{d}=38.0^{\circ} \mathrm{C} .
\end{aligned}
$$

For each combination, experiments were conducted three times and for each condition of the thermometer $(\mathrm{N}, \mathrm{A}$, and S) 30 minutes was given for the establishment of heat equilibrium inside the probes. The two probes (thin and standard types) were tested in rotation to remove the heat stored in the previous trial.

\section{Results}

3.1. Results of Simulation. In this phase, while the ambient temperature $T_{\mathrm{amb}}$ and the DBT $T_{d}$ did not change, the specific combination of these two parameters is sufficient to reveal the relations between the physical structure and the accuracy [11].

For thermometers both with and without PAR, all 24 thermometers defined by the combinations of 4 different heights and 6 different radii were considered and modeled. The measurements from each thermometer are summarized in Figure 5(a) (without PAR) and Figure 5(b) (with PAR). Markers in one interpolating curve indicate the measuring values with thermometers of the same height.

From the results, we can see that the effects of the dimensions were different. For the thermometer without PAR, generally, the accuracy was proportional to the radius, but inversely proportional to the height. A change of dimensions influences the measuring accuracy greatly when the radius is less than $40 \mathrm{~mm}$. Furthermore, the radius is more effective than the height. A twofold increase in radius benefits the accuracy much more significantly than does a half-size decrease in height.

For the thermometers with PAR, the accuracy was proportional to height and radius. It is easier for them to attain much greater accuracy with a radius greater than $20 \mathrm{~mm}$. Moreover, the height is definitive in these thermometers, as no distinct improvement can be seen by changing the radius of thermometers of the same height, with a radius larger than $30 \mathrm{~mm}$.

3.2. Results of the Mock-Up Experiment. According to the simulation results, with both standard and thin types, we can attain the acceptable margin of error $\left(<0.5^{\circ} \mathrm{C}\right)$ that is hard to attain in the designs without PAR.

In this phase, the combinations of experimental conditions (Combination 1, Combination 2, and Combination 3) were all tested and Combination 2 was the same as in the simulation phase. For both thermometers, all three conditions ( $\mathrm{A}, \mathrm{N}$, and $\mathrm{S}$ ) were tested three times each.

The results for these tests are shown in Figure 6, where the results were concluded based on the type of thermometer. In both $\mathrm{A}$ and $\mathrm{N}$ conditions, the quantitative relations conform to the results of the simulation. That is, thermometers with PAR attain better accuracy than their condition $\mathrm{N}$ counterparts. In condition $\mathrm{A}$, a thicker design gives good accuracy at the expense of a larger volume. In condition S, the measurements showed the best accuracy for both prototypes.

In condition $\mathrm{N}$, the spans of the error were about 0.5 and $0.3^{\circ} \mathrm{C}$ for standard and thin type, respectively. By contrast, the spans were about $0.2^{\circ} \mathrm{C}$ for both types in condition A. This suggests that the PAR takes effect in resisting the influence of the ambient environment. 

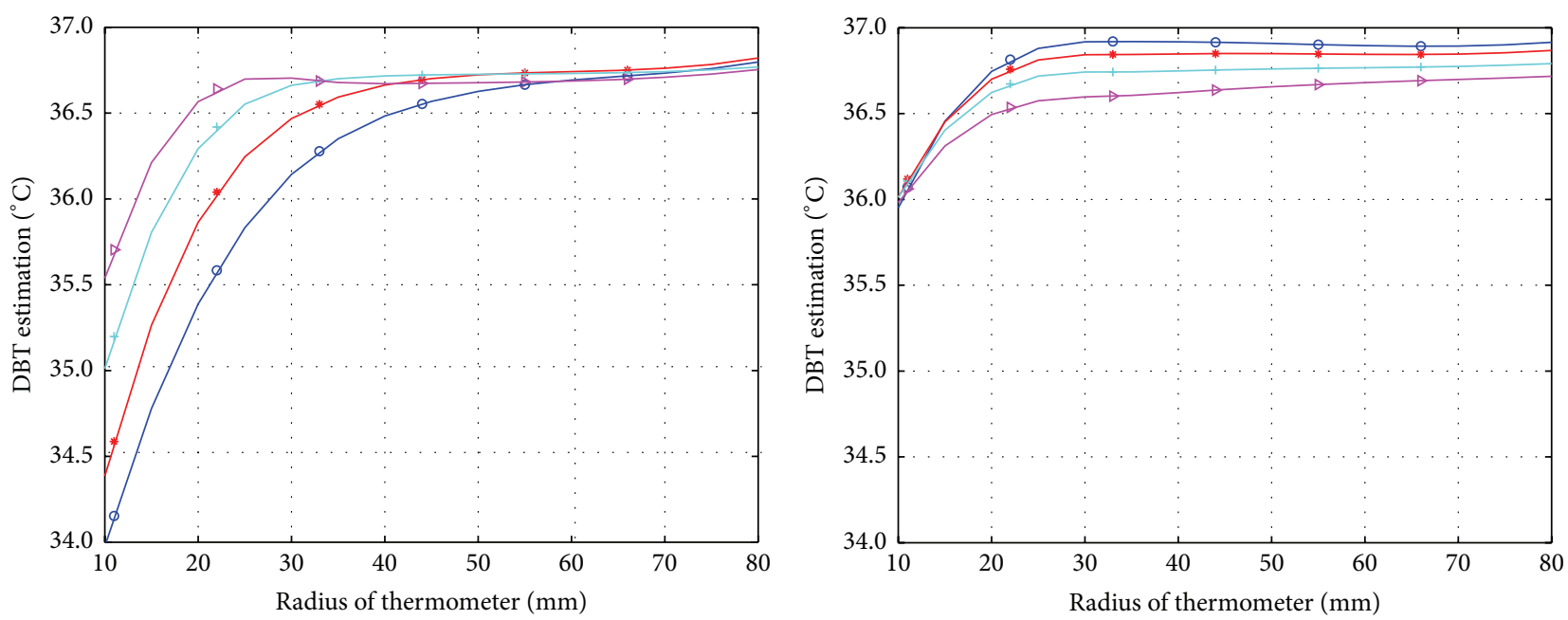

$\begin{aligned} \circ & (29,17) \mathrm{mm} \\ * & (15,9) \mathrm{mm} \\ + & (8,5) \mathrm{mm} \\ \triangleright & (4.5,3) \mathrm{mm}\end{aligned}$

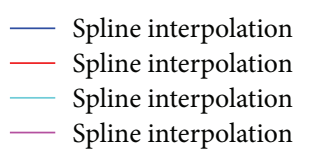

(a)

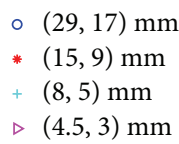

$\triangleright(4.5,3) \mathrm{mm}$

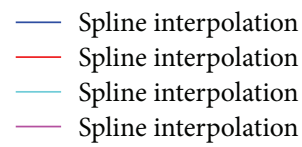

(b)

FIGURE 5: Results of simulation based on FEM. (a) and (b) show the measurements of thermometers in condition $\mathrm{N}$ and condition A, respectively.

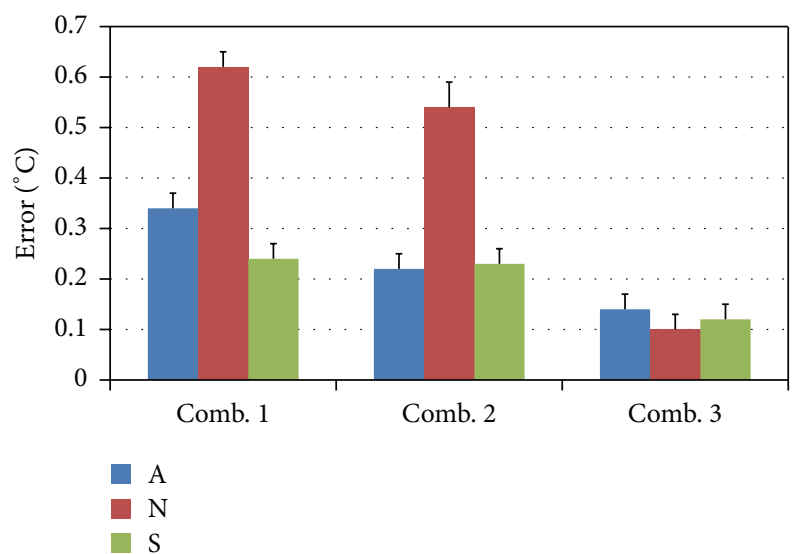

(a)
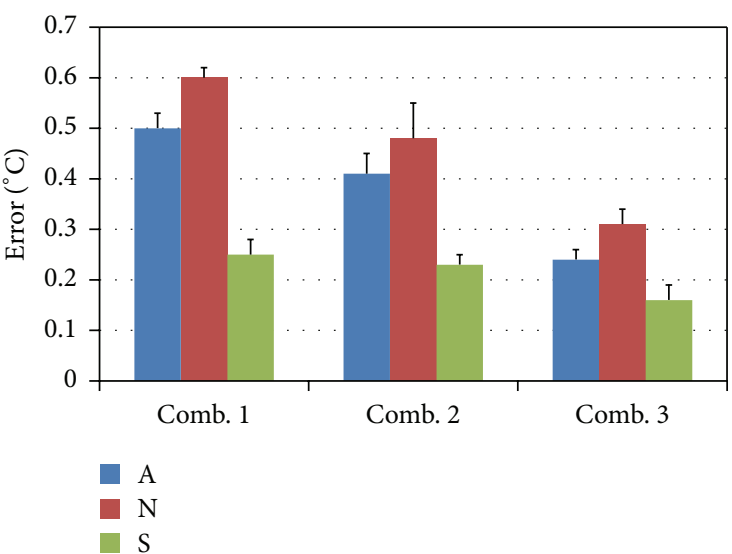

FIGURE 6: Results of mock-up experiments for both thermometers. Results of standard type were summarized in (a) and thin type in (b).

\section{Discussion}

4.1. The Practical Use. As we have introduced, the method is applicable in measuring the DBT that change at a slow manner. For extreme situation such as anesthesia or heatstroke, time delay happens. In a simulation whose results were not shown here, the standard type was used to monitor the abrupt change of DBT at a rate of $0.2^{\circ} \mathrm{C} /$ minute lasting for 10 minutes. The thermometer could indicate the change in about 4 minutes and became stable in 20 minutes after the change. For such situation, the thin type, whose reading will become stable in about 10 minutes, might be suited to trace the change.

If the thermometer is applied to the upper torso, for example, the thorax, the thermometer may come into contact with clothes from time to time. The physically thin layer and low conductivity of clothes would hardly change the resistance of the thermometer. However, the relatively high emissivity (0.75-0.90) [13] may be influential. Assuming that the thermometer comes into contact with the clothes made of cotton of $0.5 \mathrm{~mm}$ thickness for an hour, the transient study of simulation was carried out. Its result showed that the measurement of the sensor changed (decreased) after about half an hour by $0.1^{\circ} \mathrm{C}$, and then the measurement became stable gradually. In a practice, contact between the thermometer and the clothes is often erratic. In consideration of the slow and subtle change of the measurement, we considered the influence of clothes to be minor in common situation. 
4.2. The Simulation. The adoption of a PAR was based on the physical characteristics of metals, which usually have a much lower emissivity than heat insulators (e.g., rubber), and, at the same time, the radioactive energy exchange is the main reason for the horizontal heat flow distorting the practical situation from the theoretical assumption of DHFM. With a PAR, the influence of the ambient environment may be mitigated remarkably because this thermometer is designed to be worn on the torso with suitable clothing, which will shield the thermometer from the influence of airflow from concerted movement. It is also the reason why the convection was neglected in the boundary condition of the simulation.

In the simulation phase, only one pair of $T_{\mathrm{amb}}$ and $T_{d}$ was used, and the quantitative conclusions were extended to other conditions. This extension is plausible because these two factors affect the readings of the individual thermometer; however, the qualitative relations remain valid.

By comparing the results with and without PAR, distinctly different patterns of the dimensional effects (height and radius) could be seen. Further, improvements in accuracy were attained for thermometers of the same size.

In the previous study on DHFM-based thermometers [11], the radius was decisive because a larger radius means a larger area covering the skin; therefore, the heat dissipated on boundaries could be greatly compensated for by the heat from the surrounding skin. In other words, the situation will approximate the theoretical assumption of DHFM. However, with PAR, the influence of radius becomes minor, which means that less heat is demanded from the skin, resulting from less radioactive heat dissipation.

In the simulation phase, multiple sizes (height and radius) were tested, but only the $22.0 \mathrm{~mm}$ radius and the 15.0 and $9.0 \mathrm{~mm}$ heights were adopted in the fabrication stage; this limitation was imposed to retain only compact wearable sizes. An increase in radius to more than $30 \mathrm{~mm}$ gives minor improvement but will bring about difficulties in installation and an obstructive feeling.

4.3. The Mock-Up Experiment. Only 3 combinations of the ambient temperature and the DBT were investigated here. For ambient temperature, lower value results in lower accuracy due to radiation, while the relatively higher DBT would contribute to a more accurate result [11]. Therefore, Combination 1 and Combination 3 can be used to confirm the span of error under moderate environment.

In the experimental phase, condition $\mathrm{S}$ was adopted to confirm the influence of the sponge in measuring accuracy. Its positive influence on measuring accuracy may be because of the heat-insulating effect shielding the thermometer and the surrounding surface of skin from the ambient environment. However, it is actually obstructive in practical measurement and may not be suitable for wearable use.

As previously mentioned, at the mock-up experiment stage, rubber sheets of different thicknesses were tested. As we expected, the thicker the sheet, the larger the measuring error. The two prototypes were able to measure the DBT (water temperature) accurately until the thickness was increased to $8.0 \mathrm{~mm}$. The error became larger than $0.5^{\circ} \mathrm{C}$ for both thermometers when the sheet was increased to $10.0 \mathrm{~mm}$ in thickness. The main reason may lie in the abrupt decrease of the reading of sensor $T_{1}$. It may suggest that the experimental system is unable to deliver enough heat to the surface of the rubber sheet because of the lower heat conductivity of the rubber sheet [12] compared with skin. From this point of view, it is still possible for a DHFM-based thermometer to monitor the temperature about $10 \mathrm{~mm}$ under the skin. Further, to measure sites such as the forehead or the pit of the stomach with thin skin layer and bone immediately beneath [15], the measuring depth may be extended. Simulation or experiments on the measuring depth for these positions will be interesting.

For this kind of thermometer, which depends on a heat source from the human body, the resistance to the influence of ambient change is crucial. Judging from the results of these experiments, the error spans that came with the change of $T_{\text {amb }}$ were shortened considerably with this PAR.

The theory of this method suggests that $k$ here can be represented by the lengths of both heat paths. In practice, $k$ can be optimized experimentally.

With the measurements in this study, we also tried to adjust $k$, denoted by $k_{A}$ hereafter. $k_{A}$ for both prototypes was different and $k_{A}$ was set as 1.3 for the standard type and 1.1 for the thin type. For all the measurements, the accuracies were improved in varying degrees. What is more, the standard type in condition $S$ achieves the accuracy level within $\pm 0.1^{\circ} \mathrm{C}$. In the coming in vivo experiment to verify the thermometers' accuracies, adjustment of $k$ seems beneficial in attaining the best accuracies.

\section{Conclusions}

This was a preliminary study on the capability of a new kind of noninvasive DBT thermometer based on DHFM. The simulation study based on FEM brought this theory to a practical stage by proposing a new component, the PAR, to improve the accuracy and stability.

The designs were then implemented and validated through mock-up experiments. The measurement errors were mitigated to a level of less than $0.5^{\circ} \mathrm{C}$ for both designs. The standard type of $15 \mathrm{~mm}$ in height had better accuracy than the thin type, as the simulation predicted. Even though further in vivo experiments are necessary for this kind of thermometer, we believe that it can serve as an alternative to the heat-generating noninvasive DBT thermometer and is suited for wearable modality.

\section{Conflict of Interests}

The authors declare that there is no conflict of interests regarding the publication of this paper.

\section{Acknowledgments}

This study was supported by the Keihanna Science City Healthcare Project of the Ministry of Education, Culture, Sports, Science and Technology, Japan, and by Tateisi Science and Technology Foundation, Japan. The authors wish 
to thank Professor Yoshida Masaki for his kind support throughout the experiment stage.

\section{References}

[1] P. Kyriacou, Biomedical Sensors, Momentum Press, London, UK, 2010.

[2] R. H. Fox and A. J. Solman, "A new technique for monitoring the deep body temperature in man from the intact skin surface," Journal of Physiology, vol. 212, no. 2, pp. 8-10, 1971.

[3] T. Tamura, T. Nemoto, and T. Togawa, "A zero-heat-flow transducer for monitoring perfusion blood temperature," IEEE Transactions on Biomedical Engineering, vol. 11, pp. 644-646, 1979.

[4] L. P. J. Teunissen, J. Klewer, A. De Haan, J. J. De Koning, and H. A. M. Daanen, "Non-invasive continuous core temperature measurement by zero heat flux," Physiological Measurement, vol. 32, no. 5, pp. 559-570, 2011.

[5] M. Yamakage and A. Namiki, "Deep temperature monitoring using a zero-heat-flow method," Journal of Anesthesia, vol. 17, no. 2, pp. 108-115, 2003.

[6] D. S. Moran, A. Shitzer, and K. B. Pandolf, "A physiological strain index to evaluate heat stress," The American Journal of Physiology-Regulatory Integrative and Comparative Physiology, vol. 275, no. 1, pp. R129-R134, 1998.

[7] M. D. Coyne, C. M. Kesick, T. J. Doherty, M. A. Kolka, and L. A. Stephenson, "Circadian rhythm changes in core temperature over the menstrual cycle: method for noninvasive monitoring," American Journal of Physiology-Regulatory Integrative and Comparative Physiology, vol. 279, no. 4, pp. R1316-R1320, 2000.

[8] B. Bjorvatn and S. Pallesen, "A practical approach to circadian rhythm sleep disorders," Sleep Medicine Reviews, vol. 13, no. 1, pp. 47-60, 2009.

[9] H.-C. Gunga, M. Sandsund, R. E. Reinertsen, F. Sattler, and J. Koch, "A non-invasive device to continuously determine heat strain in humans," Journal of Thermal Biology, vol. 33, no. 5, pp. 297-307, 2008.

[10] K.-I. Kitamura, X. Zhu, W. Chen, and T. Nemoto, "Development of a new method for the noninvasive measurement of deep body temperature without a heater," Medical Engineering \& Physics, vol. 32, no. 1, pp. 1-6, 2010.

[11] M. Huang, T. Tamura, W. Chen, and S. Kanaya, "Evaluation of structural and thermophysical effects on the measurement accuracy of deep body thermometers based on dual-heat-flux method," Journal of Thermal Biology, vol. 47, pp. 26-31, 2015.

[12] H. H. Pennes, "Analysis of tissue and arterial blood temperatures in the resting human forearm," Journal of Applied Physiology, vol. 1, no. 2, pp. 93-122, 1948.

[13] F. P. Incropera, D. P. DeWitt, T. L. Bergman, and A. S. Lavine, Fundamentals of Heat and Mass Transfer, John Wiley \& Sons, 6th edition, 2006.

[14] T. Nemoto and T. Togawa, "Improved probe for a deep body thermometer," Medical and Biological Engineering and Computing, vol. 26, no. 4, pp. 456-459, 1988.

[15] R. Y. Ha, K. Nojima, W. P. Adams Jr., and S. A. Brown, "Analysis of facial skin thickness: defining the relative thickness index," Plastic and Reconstructive Surgery, vol. 115, no. 6, pp. 1769-1773, 2005. 


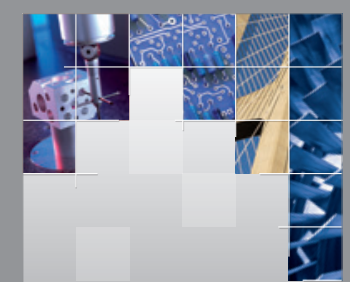

\section{Enfincering}
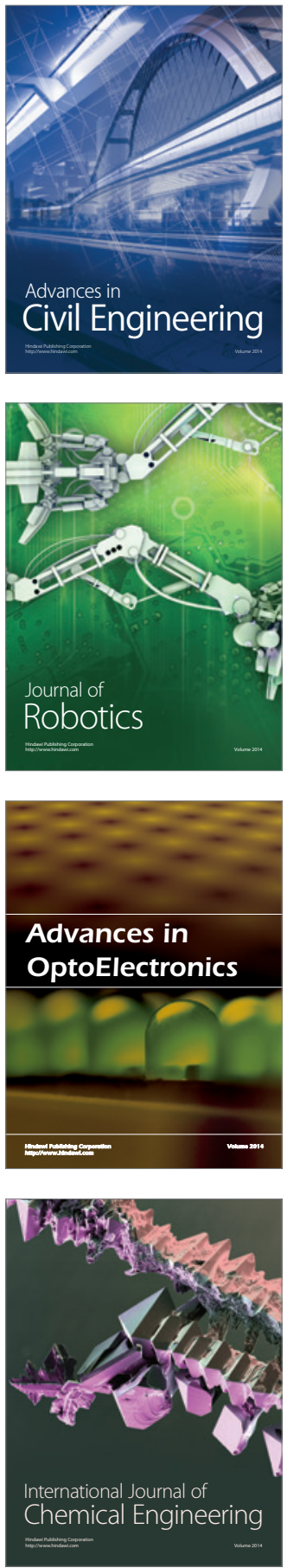

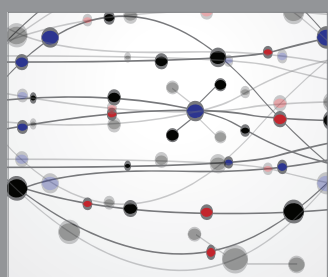

The Scientific World Journal

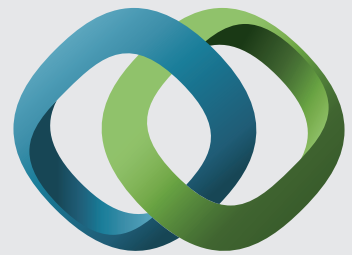

\section{Hindawi}

Submit your manuscripts at

http://www.hindawi.com
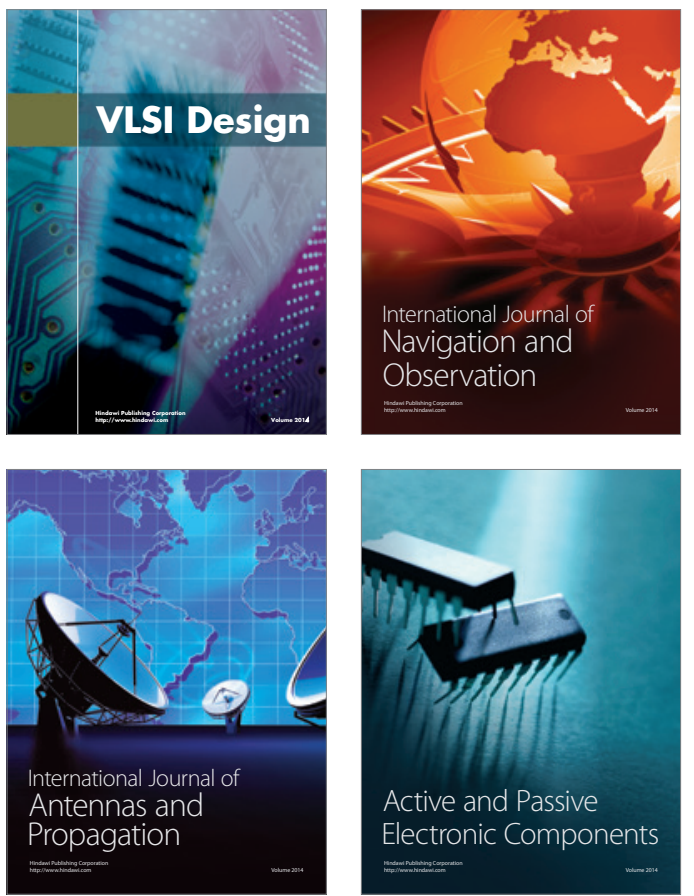
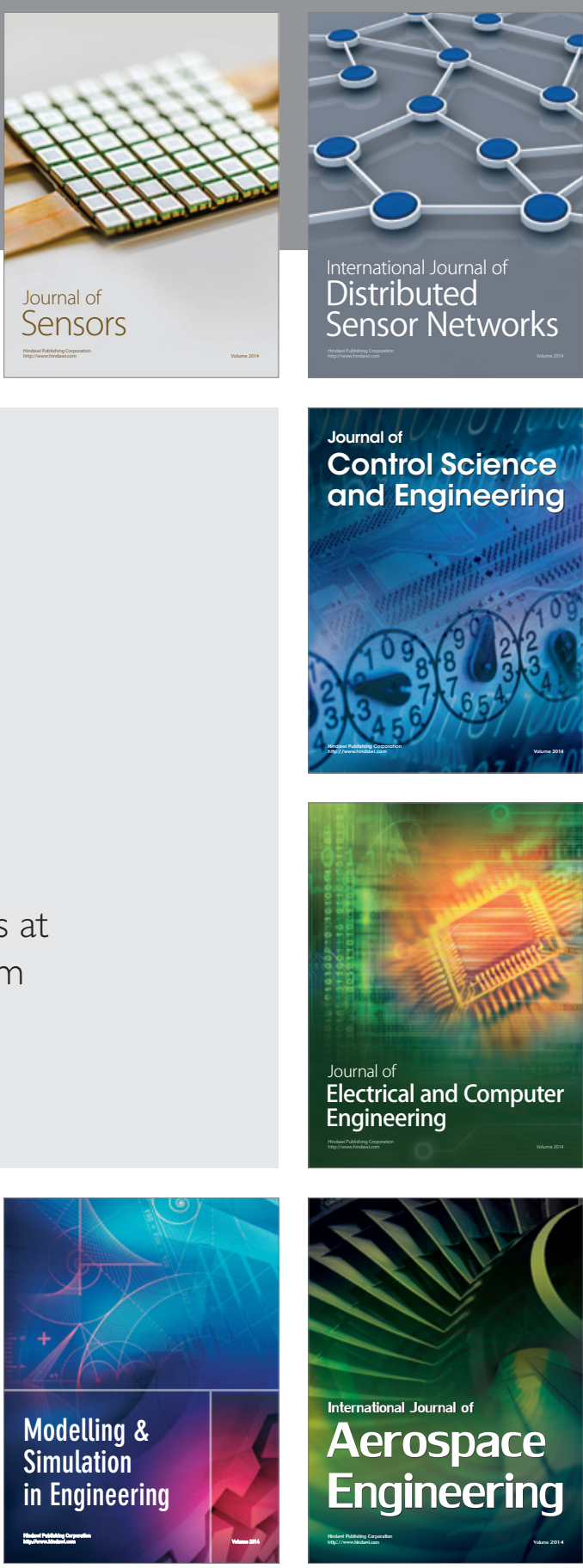

International Journal of

Distributed

Sensor Networks

Journal of

Control Science

and Engineering
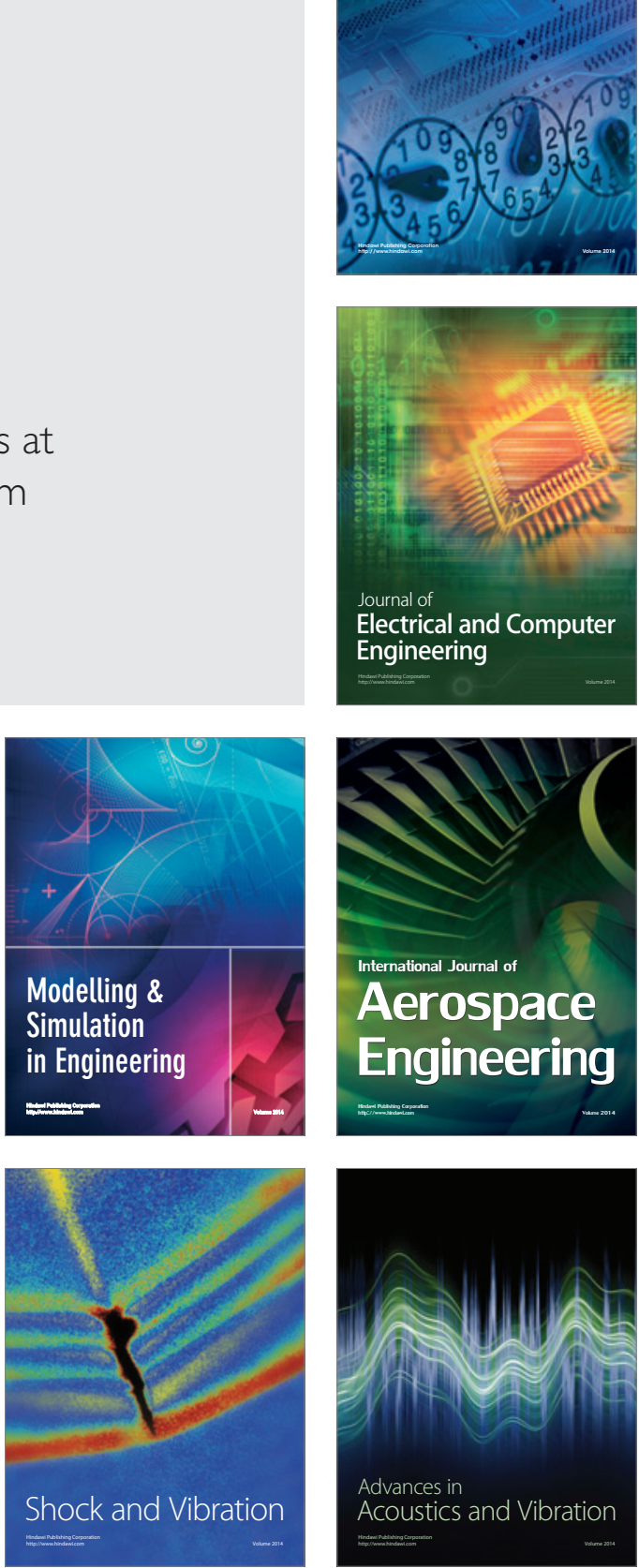